\title{
EL ALUMNADO UNIVERSITARIO Y LA PLANIFICACIÓN DE SU PROYECTO FORMATIVO Y PROFESIONAL UNIVERSITY STUDENTS AND PLANNING YOUR PROJECT AND VOCATIONAL TRAINING
}

\author{
Volumen 15, Número 1 \\ Enero - Abril \\ pp. 1-24
}

Este número se publicó el $1^{\circ}$ de enero de 2015

DOI: dx.doi.org/10.15517/aie.v15i1.16962

\author{
Pedro R. Álvarez Pérez \\ David López Aguilar \\ David Pérez-Jorge
}

\section{Revista indizada en REDALYC, $\underline{\text { SCIELO }}$}

Revista distribuida en las bases de datos:

CATÁLOGO DE LATINDEX, IRESIE, CLASE, DIALNET, DOAJ, E-REVIST@S, SHERPA/ROMEO, QUALIS, MIAR

Revista registrada en los directorios:

ULRICH'S, $\underline{\text { REDIE}}, \underline{\text { RINACE}}, \underline{\text { OEI }}$ MAESTROTECA, PREAL, $\underline{\text { CLACSO }}$ 


\title{
EL ALUMNADO UNIVERSITARIO Y LA PLANIFICACIÓN DE SU PROYECTO FORMATIVO Y PROFESIONAL UNIVERSITY STUDENTS AND PLANNING YOUR PROJECT AND VOCATIONAL TRAINING
}

\author{
Pedro R. Álvarez Pérez \\ David López Aguilar \\ David Pérez-Jorge ${ }^{3}$
}

\begin{abstract}
Resumen: En este artículo se analiza la situación del estudiantado que accede a la educación superior, a la que llegan muchas veces sin objetivos bien definidos que sirvan de guía a su proceso formativo, lo que los puede llevar a situaciones de desadaptación y deserción de los estudios. Para ello, y siguiendo una metodología de carácter descriptivo no experimental, se construyó un cuestionario que fue cumplimentado de manera anónima por una muestra de 115 estudiantes de grado de la Facultad de Educación de la Universidad de La Laguna, España. Dos grandes objetivos marcaron el desarrollo de la investigación: por una parte, analizar cómo gestiona su proceso de aprendizaje el alumnado universitario en el actual modelo educativo del Espacio Europeo de Educación Superior (EEES), y por otra, conocer qué expectativas tienen respecto a la planificación de su proyecto formativo y profesional. Los principales resultados obtenidos en el estudio pusieron de manifiesto la importancia de los procesos de información y orientación estudiantil universitaria, como elementos claves para fundamentar la toma de decisiones respecto a la planificación y el desarrollo de su carrera. Asimismo, destacó la influencia que los factores motivacionales tienen en la gestión del proyecto formativo y profesional. Como conclusión más importante, destacar la necesidad de implantar medidas orientadoras que tengan un carácter preventivo y formativo, mediante las cuales se ayude al alumnado a definir de manera continua, su proyecto formativo, y a clarificar la proyección profesional de este.
\end{abstract}

Palabras clave: ORIENTACIÓN UNIVERSITARIA, PROYECTO FORMATIVO, PLANIFICACIÓN DE LA CARRERA, RETENCIÓN ESTUDIANTIL, CALIDAD EDUCATIVA, ESPAÑA

\begin{abstract}
In this article the situation of students entering higher education is analysed. Many times, students reach this stage without clear objectives that serve as a guide to the training process, which can lead them to situations of maladjustment and school dropout. To this end, following a descriptive non-experimental methodology, a questionnaire was completed anonymously by a sample of 115 students from the Faculty of Education at the University of La Laguna (Spain). Two major goals marked the development of the research. First, analyse how university students manage their learning according to the new educational model of the European Higher Education Area (EHEA). Moreover, to know what expectations they have regarding the planning of their educational and professional project. The main results obtained in the study showed the importance of the processes of information and guidance to the university student as key elements to improve decision-making regarding the planning and career development. Similarly, it stands the influence that motivational factors have in the management of the formative and professional project. The most important conclusion highlights the need for guidance measures to help students define their educational project and clarify their professional future.
\end{abstract}

Key words: UNIVERSITY GUIDANCE, TRAINING AND PROFESSIONAL PROJECT, CAREER PLANNING, STUDENT RETENTION, EDUCATIONAL QUALITY, SPAIN

\footnotetext{
Profesor titular de Orientación Profesional. Departamento de Didáctica e Investigación Educativa. Facultad de Educación. Universidad de La Laguna. España.Dirección electrónica:palvarez@ull.es

${ }^{2}$ Becario del Plan Nacional de Formación de Profesorado Universitario (FPU). Departamento de Didáctica e Investigación Educativa. Facultad de Educación. Universidad de La Laguna. España. Dirección electrónica: dlopez@ull.es

3 Profesor Ayudante Doctor. Departamento de Didáctica e Investigación Educativa. Facultad de Educación. Universidad de La Laguna, España. Dirección electrónica:dpjorge@ull.es
}

Artículo recibido: 14 de abril, 2014

Enviado a corrección: 21 de julio, 2014

Aprobado: 20 de octubre, 2014 


\section{Introducción}

Uno de los factores que sin duda está asociado al éxito o fracaso en los estudios universitarios es el de la planificación del proyecto formativo y profesional, que tiene que servir de referencia para las diversas tomas de decisiones que a lo largo de la carrera debe enfrentar el alumnado. Sin embargo, la realidad pone de manifiesto que muchos estudiantes afrontan su proceso formativo sin una idea clara de las metas que quieren alcanzar, y sin la información adecuada acerca del perfil y de la proyección profesional que tienen los estudios que están cursando. Es decir, no tienen claro el qué y para qué de lo que hacen, y tampoco tienen claro el hacia dónde les conducirá la formación que están recibiendo, puesto que no se han identificado con metas que les haga comprometerse con su realización (Guichard, 1995; Romero, 2009). Esta falta de criterio y de argumentos para construir su proyecto formativo y profesional, conduce en muchas ocasiones a situaciones de inseguridad, improvisación, desmotivación, etc., y en el peor de los casos, al abandono de la formación.

Precisamente, el fracaso y abandono de los estudios es uno de los principales problemas que afecta a la institución universitaria y aumentar la persistencia y frenar la deserción uno de los objetivos prioritarios que se intenta conseguir a nivel mundial (Taylor y Millar, 2002; Ryan y Glenn, 2003). Las investigaciones realizadas hasta el momento (Álvarez, Cabrera, González y Bethencourt, 2006; Álvarez, Figuera y Torrado, 2011; Cabrera, Bethencourt, González y Álvarez, 2006; Elias-Andreu, 2008; Gairín, Figuera y Triadó, 2010) evidencian la relevancia del problema, dados los elevados índices que se vienen registrando, que en algunos casos superan hasta el $50 \%$ en los dos primeros años de formación, lo que pone de manifiesto lagunas importantes y fallos en el sistema, que hacen necesario una revisión de los factores implicados en el proceso formativo del alumnado. En la Universidad de La Laguna, el estudio llevado a cabo por González, Álvarez, Cabrera y Bethencourt (2007) con una muestra de 163 estudiantes de tres carreras diferentes (Pedagogía, Matemáticas y Trabajo Social), determinó que el 28\% abandonan los estudios en los dos primeros años de carrera, solo el $20 \%$ termina su formación en el tiempo prescrito por la carrera y el $52 \%$ logra también concluir sus estudios, pero prolongando el número de años en la universidad. El problema no concluye en las instituciones educativas, sino que su proyección al ámbito social y económico es evidente. Aproximadamente, el $70 \%$ del desempleo en jóvenes menores de 25 años tiene una estrecha relación con el fracaso 0 abandono de los estudios. 
Todos estos factores forman parte del conjunto de problemas de tipo personal, vocacional y profesional que, ligados a la nueva realidad socioeconómica que se vive actualmente, determinan la actitud de los jóvenes hacia la vida en general y hacia la formación en particular y condicionan su compromiso y responsabilidad de cara a estructurar su proyecto personal, del que una parte importante se configura durante el tiempo en el que permanecen en el sistema educativo (Castro y Sánchez, 2000; Mendías y Camacho, 2004; Tejada, 2003). De este modo, la concatenación de decisiones afecta a la dirección del proyecto formativo y profesional, ya que el paso por la enseñanza ejerce una influencia y marca la tendencia hacia el logro de unas metas concretas, lo que determinará luego todo el recorrido vital. Si el paso por el sistema educativo se afronta desde la improvisación y sin una conciencia clara de la influencia que la falta de planificación tiene en los avances futuros, es posible que muchos de los jóvenes, no solo abandonen el sistema educativo con una formación deficitaria, sino que tendrán menos posibilidades y mayores dificultades para integrarse en la vida sociolaboral activa y en el mundo del trabajo (Davia, 2004; Figuera, Torrado y Dorio, 2011). Por eso, como señalan Santana y Feliciano (2009), el sistema educativo tiene que asumir el reto de impulsar la orientación académica y profesional de los jóvenes, para que logren un adecuado conocimiento de sí mismo, mejoren sus habilidades para tomar decisiones y sean capaces de realizar una correcta planificación racional de los diferentes itinerarios formativos que les lleven a alcanzar sus metas laborales.

El presente se nutre de las experiencias vividas por los jóvenes, de las aspiraciones y expectativas, de los proyectos que construyen, de la exploración de las distintas opciones a partir de las cuales trazan una línea a seguir para alcanzar unos objetivos, que en la mayoría de los casos tiene que ver con la inserción laboral. Por eso, en la realidad actual hay que salvar el desánimo que en ocasiones impera en los jóvenes estudiantes cuando piensan en su futuro académico profesional y que deriva en muchos casos hacia el absentismo (Álvarez y López, 2011), la falta de asistencia a clase (Álvarez et al., 2004), la desmotivación por la enseñanza (Gámez y Marrero, 2003), o la falta de expectativas hacia el mundo laboral (Santana, 2001). El estado de incertidumbre que afecta a la actual generación de jóvenes, que en un periodo corto de tiempo tendrán que afrontar el acceso al mundo del trabajo, se debe combatir desde la certeza de que ahora más que nunca, tiene que primar la acción personal, la responsabilidad consigo mismos a la hora de llevar a cabo las elecciones vocacionales, la necesidad de prepararse para afrontar los cambios a lo largo de la vida y la definición de trayectorias e itinerarios ajustados a las posibilidades e intereses de cada uno 
(Álvarez, 2002; Hernández, 2004; Monge, 1993; Rivas, 1990 y Rodríguez, 1998). Desde esta perspectiva, la construcción del proyecto formativo y profesional tiene que ser la herramienta personal para liberar ese futuro hipotecado y condicionado por el presente (Gallego, 1999; Romero, 2004).

Esta situación es especialmente relevante en el modelo educativo del Espacio Europeo de Educación Superior (EEES), donde el alumnado debe demostrar autonomía en el proceso de aprendizaje y capacidad para gestionar su proceso formativo a lo largo de la vida, para lo que requiere el dominio de una variedad de competencias necesarias, no solo durante su paso por la educación superior, sino al transitar al mundo del trabajo (Alonso, 2004). Y entre dichas competencias, no cabe duda de que la capacidad de organización y planificación constituye un factor de primer nivel que el alumnado debe adquirir y desarrollar (Blanco, 2009; Villa y Poblete, 2010).

En este nuevo escenario formativo, a diferencia de etapas previas en las que el alumnado se dejaba llevar por la inercia del curso a curso, el estudiante tiene que asumir el protagonismo de su proyecto formativo profesional. Y para ello, como señala Corominas (2006), tiene que conocer con claridad las características y condiciones en las que se efectuará el proceso de aprendizaje y activar todas las estrategias para incorporarse, obtener un buen rendimiento académico y definir la proyección profesional que le quiere dar a sus estudios.

Esta nueva realidad obliga a intensificar la orientación al alumnado, sobre todo en estos primeros momentos de su andadura por la educación superior (Corominas e Isus, 1998; Echeverría, 2008; Martínez, 2009; Sánchez et al., 2008). Por eso, con la implantación del Espacio Europeo de Educación Superior, se han potenciado las medidas de orientación al alumnado y en el Estado español, tanto el Real Decreto 1393/2007 (corregido posteriormente por el Real Decreto 861/2010) por el que se ordenan las enseñanzas universitarias oficiales, como el Real Decreto 1791/210 del Estatuto del Estudiante Universitario, reconocen la importancia de la orientación en el contexto universitario, como un elemento clave para la garantía de la calidad y la acreditación y obligan a los centros universitarios a planificar sistemas de información previa a la matriculación, procedimientos de acogida y orientación de la población estudiantil de nuevo ingreso y sistemas de apoyo y orientación a lo largo de su carrera.

Asimismo, se le ha dado una consideración diferente al rol profesional del profesorado, ya que a la función de transmisor de contenidos científicos, se ha unido ahora la de 
facilitador del aprendizaje autónomo y de acompañante, durante el recorrido por los diferentes tramos formativos, lo que implica el desempeño de la función tutorial y el seguimiento del proceso de aprendizaje estudiantil (Arenas, Bilbao, Rodríguez y Jiménez, 2003). Al respecto, señala Salinas (2000), que el profesorado universitario tiene que prepararse para un nuevo rol que actúe como guía y facilitador de recursos que orienten a estudiantes que participen en su propio proceso de aprendizaje.

Para avanzar en el desarrollo de este modelo formativo que propugna el Espacio Europeo de Educación Superior, y en el perfil de profesor tutor universitario que se reclama, será necesario mejorar la formación que tienen los docentes en relación con el desempeño de la función tutorial. Como señalan Sola y Moreno (2005, p. 133), "la falta de formación del profesorado en materia de tutoría y la carencia de hábitos de actuación sistemática en orientación educativa, genera una situación poco optimista a los resultados del ejercicio de la acción tutorial". Para corregir estas deficiencias sería necesario implantar programas de formación para los profesores tutores (Coriat y Sanz, 2005), que faciliten la introducción de la acción tutorial como un componente de la labor educativa del profesorado, de modo que contribuyan a la formación integral del alumnado y al desarrollo de las distintas competencias, tanto de carácter académico como profesional.

Y uno de los ámbitos que se debería reforzar desde el espacio de la tutoría universitaria, es el de la información y la ayuda para lograr una buena adaptación y planificación de su proyecto personal, de modo que los estudiantes afronten con responsabilidad y con proyección su proceso de aprendizaje y enseñanza (Álvarez, 2012; Martín, Moreno y Padilla, 1998; Romero, 2003; Vieira, 2008). Por eso, en este estudio intentamos profundizar en el modo en el que los estudiantes gestionan su proceso formativo, examinando qué visión tienen de la titulación que cursan, cuál es su grado de implicación y compromiso con la formación, y en qué medida tienen claras sus metas y proyectos de futuro.

\section{La planificación de la carrera y el riesgo de fracasar en los estudios}

Uno de los objetivos importantes que se vienen planteando desde el ámbito de la orientación universitaria, es ayudar a los jóvenes a adaptarse al contexto de los estudios superiores, planificar el desarrollo de su carrera y definir su proyecto formativo y profesional (Cano, 2009). Se trata de asesorar al estudiantado en el esclarecimiento de sus posibilidades y en la construcción de un plan de desarrollo realista, ajustado a sus 
condiciones personales y de contexto. El proyecto formativo y profesional forma parte del proyecto vital de cada persona, y representa el sentido que cada uno quiere darle a su vida en relación con el desarrollo profesional y el desempeño de determinados roles que considera relevantes. Así, las decisiones que se tomen en las distintas transiciones vitales, deben vincularse con este proyecto vital. Romero (2004, p. 338) define el proyecto profesional y vital como:

la construcción activa en la que se trabaja sobre y se expresa la propia imagen de sí mismo, que implica la adquisición de una serie de conocimientos, habilidades y actitudes, que requieren de un proceso previo de información/exploración/formulación de objetivos, que se concreta en un plan de acción, que genera desarrollo personal y que puede ser aprendido.

Por tanto, si hay que destacar una característica del sentido que tiene el proyecto formativo y profesional estudiantil, es la anticipación. Rodríguez (2003) plantea la necesidad de afrontar la definición de los proyectos personales desde una perspectiva circular, cíclica, intencional y analítica, trazando una línea de continuidad entre la percepción del presente y lo que cada uno considera que debería ser el futuro. Desde esta concepción del proyecto formativo y profesional, el estudiantado tiene que adoptar una actitud de continuo cuestionamiento y de planificación de los pasos que permiten darle forma a su proyecto. Entre el "ahora" y el "después", media toda una definición de metas personales y profesionales, y la clarificación de la identidad del sujeto, que crece desde la autonomía y la consolidación de los pasos que va recorriendo, durante el tiempo en el que pone en práctica su plan de acción orientado al logro de metas. En este sentido, el estudiantado ha de llevar a cabo una labor prospectiva acerca de su futuro, entendida como una acción de construir a partir de las experiencias previas, pero con un sentido de mirar hacia delante. Como dice Rodríguez (1998, p. 257), al referirse al proyecto profesional y vital

es acción y una reflexión de triple orden: sobre la situación presente, sobre el futuro que se desea y sobre los medios que se van a precisar para conseguirlo (...) a partir de este planteamiento se han de reevaluar y redefinir los objetivos y metas hacia las que la persona apunta.

Este proceso de definición progresiva, de ordenación y acción intencional, debe caracterizar la construcción del proyecto formativo y profesional de los jóvenes, ya que las 
respuestas a los problemas con los que se enfrenta de forma progresiva a lo largo de su vida, así como su capacidad para adaptarse a las nuevas situaciones de forma madura y satisfactoria, dependerá en gran parte del repertorio de aprendizajes y de conductas aprendidas. Frente a la actitud pasiva con la que muchos estudiantes afrontan su educación universitaria, el carácter dinámico, evolutivo y procesual con la que se debe afrontar la construcción del proyecto formativo y profesional, hace necesario un posicionamiento de continua búsqueda, una actitud ante los diversos cambios que se suceden, un posicionamiento desde determinados valores y objetivos, ante las oportunidades que cada uno descubre, una búsqueda de la autonomía personal para superar los obstáculos y dar forma y consolidar un itinerario de desarrollo personal y profesional (Álvarez, Figuera y Torrado, 2011; García, 2008).

Por el contrario, la falta de perspectivas, el vacío intencional, motivacional y emocional con el que se afronte el proceso de aprendizaje, pueden ser causas suficientes para no superar los obstáculos y para dejar la universidad sin haber conseguido antes la preparación que les capacite para el mundo del trabajo. Como señalan García, Asensio, Carballo, García y Guardia (2005, p. 200) "la actitud negativa hacia la institución educativa puede derivar hacia el absentismo, abandono y búsqueda de otros objetivos y valores externos a la universidad y la sociedad". Estaríamos ante una cohorte de estudiantes cuya preparación para afrontar los cambios durante los estudios universitarios es inapropiada, dado que ni poseen la información necesaria, ni han planificado los pasos que deben recorrer en su proyecto personal, ni han desarrollado las habilidades para hacer frente a las diversas situaciones, ni poseen la autonomía, la capacidad de decisión, la capacidad de adaptarse a nuevas situaciones, etc., que requiere la gestión de su desarrollo personal, académico y profesional (Martínez, 2003; Taveira y Rodríguez, 2010).

El estudiantado que presenta estas características tendrá muchas dificultades para adaptarse al nuevo modelo formativo del EEES, puesto que entre sus señas de identidad cabe destacar el papel activo que se le reclama al alumnado, que ha de ser el principal actor del proceso de aprendizaje, la necesidad de definir itinerarios formativos con proyección al desarrollo profesional, el énfasis en el aprendizaje autónomo, el desarrollo de competencias, o el aprendizaje a lo largo de la vida. Como señalan García, et al. (2005) y García y Salmerón (2010), el nuevo EEES y la universidad actual demandan un perfil de estudiantes universitarios con capacidad de planificar su proyecto formativo y profesional, con capacidad para gestionar su carrera, autónomo, implicado en su proceso de aprendizaje, motivado por 
el estudio y con capacidad para seguir aprendiendo a lo largo de la vida. Por eso, el aprendizaje permanente y la aplicabilidad de las competencias en distintos momentos y situaciones de la vida de cada uno, se ha convertido en uno de los ejes claves del nuevo enfoque de la educación superior y uno de los objetivos importantes que la institución universitaria debe alcanzar (Peter, 2006; Zabalza, 2003).

En la definición y gestión de su proyecto personal, interviene la motivación que alimenta este proceso y que a su vez se relaciona con la toma de decisiones y la selección de objetivos y metas. Todos estos factores correlacionan con el rendimiento, ya que tienen una influencia alta en la manera en la que el estudiantado afronta las tareas de aprendizaje, el compromiso y la responsabilidad con el que se implican en la resolución de problemas y la persistencia para lograr los objetivos personales de su proyecto formativo y profesional (Castro y Sánchez, 2000). Es decir, la claridad de metas que dan forma al proyecto personal de cada uno, lo predispone para un enfrentamiento constructivo con el proceso de aprendizaje. De ahí se podría deducir que quienes poseen un proyecto formativo y profesional, estarán más implicados con el proceso de aprendizaje que quienes estén desinteresados e indiferentes.

En relación con esta temática giró el estudio que hemos realizado, en el que se abordó cómo gestiona el estudiantado universitario su proceso formativo y en qué medida se preocupan por definir su proyecto personal durante el tiempo que dura la carrera. Concretamente, los objetivos que guiaron la investigación fueron:

1. Conocer la información que tenían los estudiantes universitarios antes de acceder a la enseñanza superior.

2. Conocer la valoración, expectativas y motivación que tenían los estudiantes al incorporarse a la formación universitaria.

3. Analizar los procesos de planificación del proyecto personal que llevan a cabo los alumnos universitarios a lo largo de su proceso formativo.

4. Identificar las metas y proyectos de futuro que tienen los estudiantes universitarios.

5. Conocer la relación entre la información sobre la carrera y servicios de la universidad y la valoración que el alumno atribuye a la carrera que elige. 


\section{Método}

\subsection{Participantes}

Los participantes de este estudio fueron estudiantes de las carreras de Grado de Maestro de Educación Infantil y Grado de Maestro de Educación Primaria de la Facultad de Educación de La Universidad de La Laguna. Concretamente, el requisito que debían cumplir los participantes era el ser estudiantes universitarios de los dos primeros cursos de estas carreras. Con ello se aseguraba la posibilidad de hacer una valoración de la trayectoria académica seguida por el estudiantado hasta la enseñanza universitaria, una valoración de cómo afrontaban el tránsito y adaptación a la educación superior y cómo planificaban los pasos de su itinerario académico y profesional.

De la población total de 687 estudiantes, y asumiendo un nivel de confianza del 95,0\%, con un margen de error del $\pm 9,0 \%$, se estimó que se debía contar con la participación de al menos 102 estudiantes que reunieran los requisitos descritos. A partir de un muestreo de conveniencia, la muestra quedó conformada por 115 estudiantes, lo que permitió trabajar con un intervalo de confianza del $95,0 \%$ y un margen de error del $\pm 8,34 \%$. La distribución de la muestra se situó en un rango de edades comprendido entre 18 y 56 ( $\bar{x}=22,16 ; S d=5,46)$. En cuanto al género, el $51,03 \%$ ( $n=59)$ eran mujeres y el $48,69 \%(n=56)$, hombres.

\subsection{Instrumento de recogida de datos}

La estrategia utilizada para la recogida de datos fue la elaboración de un cuestionario ad hoc adaptado a los objetivos del estudio realizado. Concretamente, se empleó un cuestionario para aplicar de manera presencial denominado "Cuestionario de Planificación del Proyecto Formativo y Profesional del Alumnado Universitario", que fue respondido de forma voluntaria y anónima, durante las sesiones presenciales de clase.

Previo a la aplicación definitiva de la prueba, se realizaron procedimientos de validación, con la finalidad de garantizar el rigor y la adecuación del instrumento a los propósitos de la investigación. Por una parte, se sometió la primera versión del cuestionario a una prueba de pilotaje para depurar algunos aspectos referidos a la redacción, contenido, compresión de ítems, opciones de respuesta, tipo de preguntas, etc. Para ello, se aplicó la prueba en una sesión presencial a 7 personas que reunían similares características a las de la muestra. Durante la prueba, 3 evaluadores recogieron todas las incidencias relacionadas con los aspectos descritos. Por otra parte, 4 docentes universitarios especialistas en el ámbito de la educación efectuaron una prueba de contenido. El objetivo era valorar aspectos 
referidos a la estructura, la pertinencia, la claridad y adecuación de las preguntas, el contenido, etc.

Después de introducir las correcciones oportunas a partir de las pruebas realizadas y tal como se muestra en la tabla 1, se configuró la versión definitiva del cuestionario, en la que se agruparon los ítems en torno a 4 dimensiones centrales determinadas para este estudio: (información antes de entrar a la universidad (I), adaptación a los estudios universitarios (II), planificación del proceso formativo (III) y metas y proyectos de futuro (IV)). La prueba final contenía un total de 35 preguntas distribuidas en escalas de valoración (1-4) (ítems 1, 4, 9, 10, 12, 13 18, 22 y 26), preguntas dicotómicas con preguntas abiertas (ítems $2,5,7,11,16,17,23,24,25,27,30,32,33,34$ y 35), preguntas de respuesta múltiple (ítem $6,8,19,20,21$ y 28 ) y preguntas de respuesta abierta (ítems $3,14,15,29$ y 31 ).

Tabla 1. Distribución de ítems, según dimensiones de la prueba

\begin{tabular}{|c|c|}
\hline Dimensiones & Ítems \\
\hline Información antes de entrar a la universidad & $1,2,3,4,5,6$ y 7 \\
\hline Adaptación a los estudios universitarios & $8,9,10,11,12,13$ y 14 \\
\hline Planificación del proceso formativo & $15,16,17,18,19,20,21,22,23,24,25,26,27,28,29$ y 30 \\
\hline Metas y proyectos de futuro & $31,32,33,34$ y 35 \\
\hline
\end{tabular}

Fuente: elaboración propia

El coeficiente de fiabilidad del instrumento definitivo se obtuvo mediante la aplicación de la prueba alfa de Cronbach a los 37 ítems que configuraban las escalas tipo Likert utilizadas en el cuestionario (preguntas 1, 9, 22 y 26). El valor obtenido ( $\alpha=.820$ ) indicó una alta consistencia interna y una interrelación y nivel de estabilidad significativo.

\subsection{Análisis e interpretación de los datos}

Dado que el objetivo principal de la investigación era recoger información que permitiera valorar cómo planifican y gestionan su proyecto formativo y profesional el estudiantado universitario, se llevó a cabo, por un lado, un análisis descriptivo de las respuestas a las preguntas del cuestionario, y por otro, un análisis comparativo que permitiera visualizar los patrones de relación entre aquellas variables que mostraron una distribución conjunta de frecuencias significativas (4-8; 4-10; 8-10; 8-15; 10-15).

Para ello, una vez administrado el instrumento, los datos fueron analizados a través del programa Statistical Package for the Social Sciences (SPSS 17.0) para el sistema operativo 
Microsoft Windows 7. De manera específica, la prueba empleada fue el estadístico jicuadrado, como prueba de interdependencia de variables categóricas. Para las preguntas abiertas se utilizó la técnica de análisis narrativo, categorizando las respuestas de cada uno de los ítems.

\section{Resultados}

Los resultados de este estudio se presentan organizados en función de las dimensiones propuestas en la Tabla 1.

\section{1 Información antes de entrar a la universidad}

En esta primera dimensión, se analizó la información que tenían los estudiantes antes de entrar en la universidad. Los datos recogidos evidencian que el alumnado no disponía de información sobre: las salidas profesionales de las carreras universitarias (73,2\%), los servicios e instalaciones de la universidad (86,6\%), las ayudas y becas de las que disponían $(72,1 \%)$, sus derechos y deberes $(88,4 \%)$ y los programas de apoyo y orientación para el estudiantado universitario (91,9\%) (Gráfico 1).

Gráfico 1. Información sobre estudios universitarios

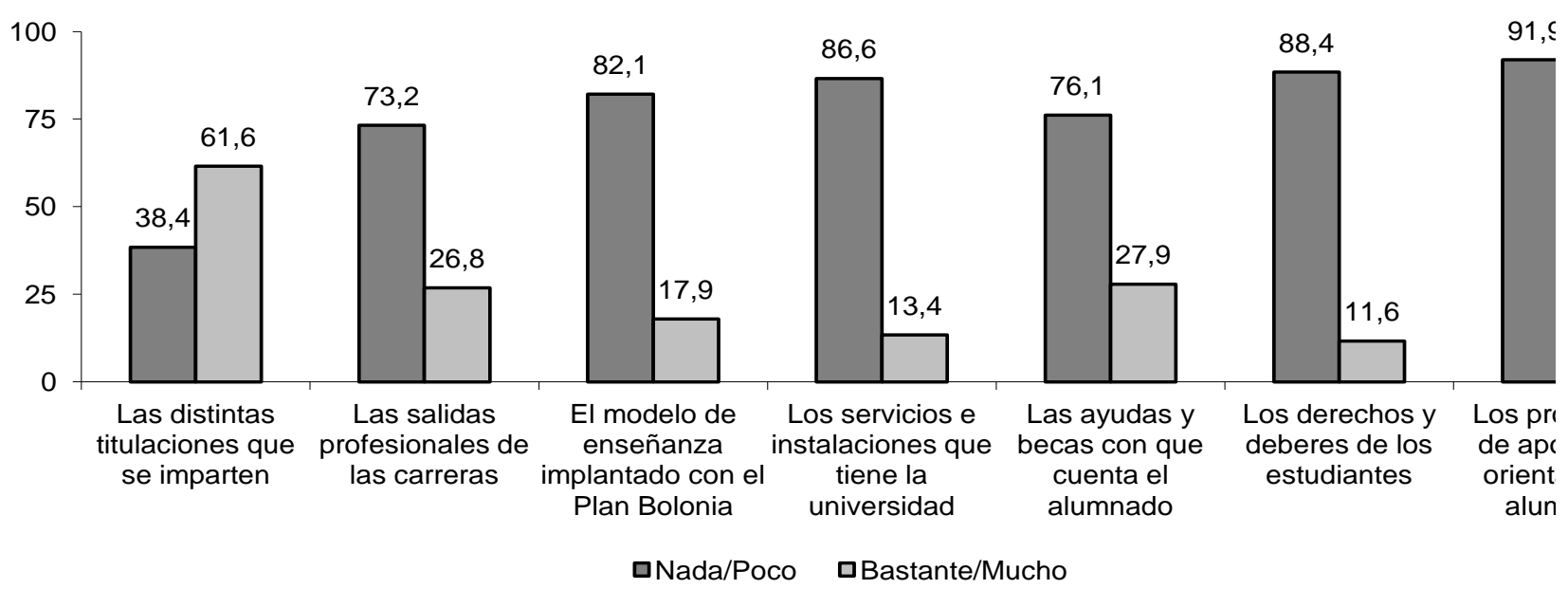

Fuente: elaboración propia

Uno de los elementos que influye en los procesos de toma de decisiones académicas tiene que ver con la información que tienen los estudiantes acerca de las distintas opciones formativas a las que pueden acceder. En este sentido, destacaba que tan solo el $47,8 \%$ 
manifestó tener bastante / mucha información relacionada con los estudios que pretendía realizar. Sin embargo, y pese a este escaso conocimiento de las carreras, el 87,6\% de los estudiantes consideró tener definido el proyecto académico-profesional que pretendía desarrollar.

En cuanto a las razones que tuvieron en cuenta para elegir los estudios universitarios, destacaron: el gusto por la carrera (93,9\%), la adecuación de los estudios a la capacidad y características personales del estudiante (63,5\%), y las salidas profesionales del título universitario $(30,4 \%)$.

\subsection{Adaptación a los estudios universitarios}

En relación con este apartado, el 71,9\% del alumnado señaló tener un nivel alto de motivación en el momento de iniciar sus estudios universitarios. Lo confirmó el hecho de que el $77,5 \%$ del estudiantado no se planteó abandonar los estudios porque le gustaba la carrera que estaba cursando. En las respuestas abiertas, muchos de los participantes incidieron en la satisfacción con los estudios elegidos: "porque me gusta" (p3), "porque me gusta y me satisface" (p17), "porque me gusta lo que estudio" (p51), "porque estudio algo que me gusta" (p83), y por las salidas profesionales: "por motivos laborales" (p19) y "porque me gusta la profesión" (p57).

Sin embargo, la motivación variaba en los casos en que el estudiantado permanecía en la carrera. Si bien el 40,9\% indicó que mantenía la expectativa que tenía hacia la carrera que cursaban, el 37,4\% la había empeorado y solo el 21,7\% manifestó que su valoración acerca de la carrera que cursaba había mejorado, indicando en estos casos que "seguiría en esta carrera" (p61), "volvería a matricularme" (p74) o "haría la misma" (p101).

Uno de los factores que determina la adaptación a la enseñanza universitaria tiene que ver con el grado de información sobre la carrera y el conocimiento sobre los servicios y recursos que ofrece la universidad (ítems 4-8). En este sentido, los estudiantes que tenían bastante o mucho conocimiento previo de la universidad, tenían unas expectativas más precisas y realistas sobre la carrera que pretendían cursar, mientras que aquellos que manejaban nada o poca información, se encontraron con unos estudios que no se ajustaban a sus motivaciones académicas previas (Tabla 2). 
Tabla 2. Análisis de correspondencia entre información sobre la carrera y valoración de esta

\begin{tabular}{|c|c|c|c|}
\hline \multirow{2}{\text{ITTEM4:Informaciónsobrela}}{$\begin{array}{c}\text { ITEM 8: Valoración de la carrera } \\
\text { carrera y servicios de la } \\
\text { universidad }\end{array}$} & $\begin{array}{c}\text { Tal y como me la } \\
\text { esperaba }\end{array}$ & $\begin{array}{c}\text { Peor de lo que me } \\
\text { esperaba }\end{array}$ \\
\cline { 2 - 4 } & Mejor de lo que esperaba & 0,200 & $\mathbf{0 , 8 0 0}$ \\
\hline Ninguna & 0,000 & 0,327 & 0,418 \\
\hline Poca & 0,255 & 0,457 & 0,326 \\
\hline Bastante & 0,217 & $\mathbf{0 , 7 7 8}$ & 0,111 \\
\hline Mucho & 0,111 & 0,409 & 0,374 \\
\hline Peso & 0,217 & & \\
\hline
\end{tabular}

Fuente: elaboración propia

La relación entre la información y la valoración de la carrera no resultó significativa $(p=0,08)$. Sin embargo, se observa que el análisis de correspondencias muestra que la dimensión 1 es la que mejor permite separar las categorías de respuesta para el ítem 4 en cuanto al ítem 8 (figura 1). Esto se traduce en un porcentaje de varianza explicado por los ítems de información sobre la carrera y servicios de la universidad, fue del $78 \%$ frente al $21 \%$, que hizo referencia a la valoración que hacen los estudiantes acerca de los estudios universitarios que cursan. Este dato muestra la utilidad que tiene la información acerca de la carrera y los servicios que ofrece la universidad, en la explicación de la nube de puntos y la relación existente entre el conocimiento previo y la valoración de la carrera.

Figura 1. Relación entre información sobre la carrera universitaria y valoración de esta

\section{Row and Column Points}

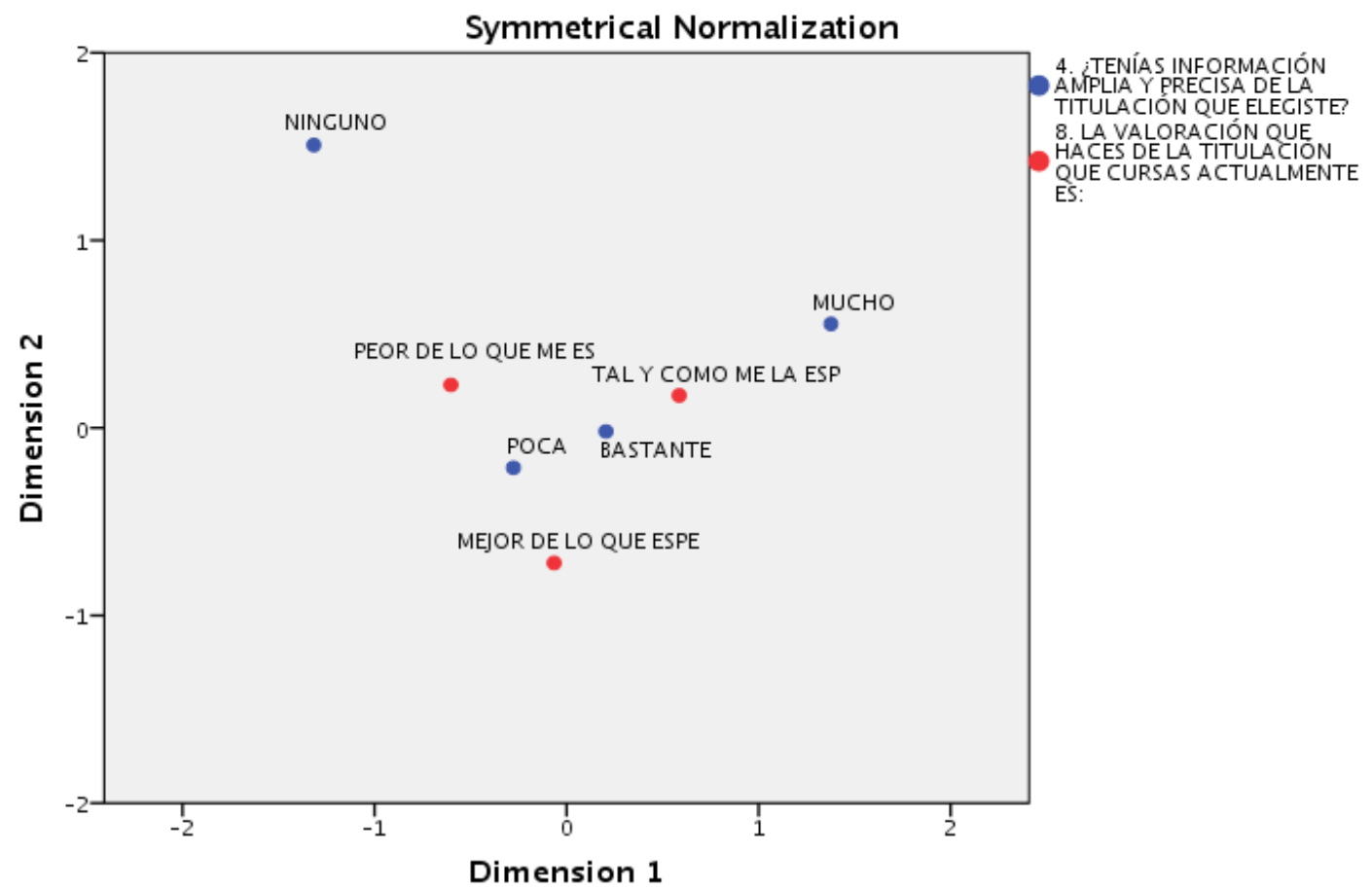

Fuente: elaboración propia 
Cabe resaltar la relación significativa encontrada entre la información previa y la motivación durante los estudios (ítems 4-10), dado que las respuestas están vinculadas $(p<0,05)$. De esta manera, los estudiantes que no disponían de información previa al momento de acceso a la enseñanza universitaria, mostraron un bajo nivel de motivación, mientras que los que se habían preocupado por tener conocimiento e información sobre la carrera, los servicios y recursos que ofrece la universidad, se identificaban con una motivación buena o excelente.

Otros aspectos que destacaron los estudiantes en este cambio de expectativas hacia los estudios universitarios, tenían que ver principalmente con distintos elementos relacionados con los procesos formativos de la educación superior, tales como la metodología desarrollada por el profesor, el nivel de exigencia, el contenido que se trata en las diferentes materias y las prácticas que se realizan a lo largo de su formación (Gráfico 2).

Gráfico 2. Elementos que condicionan el cambio de expectativas hacia la formación universitaria.

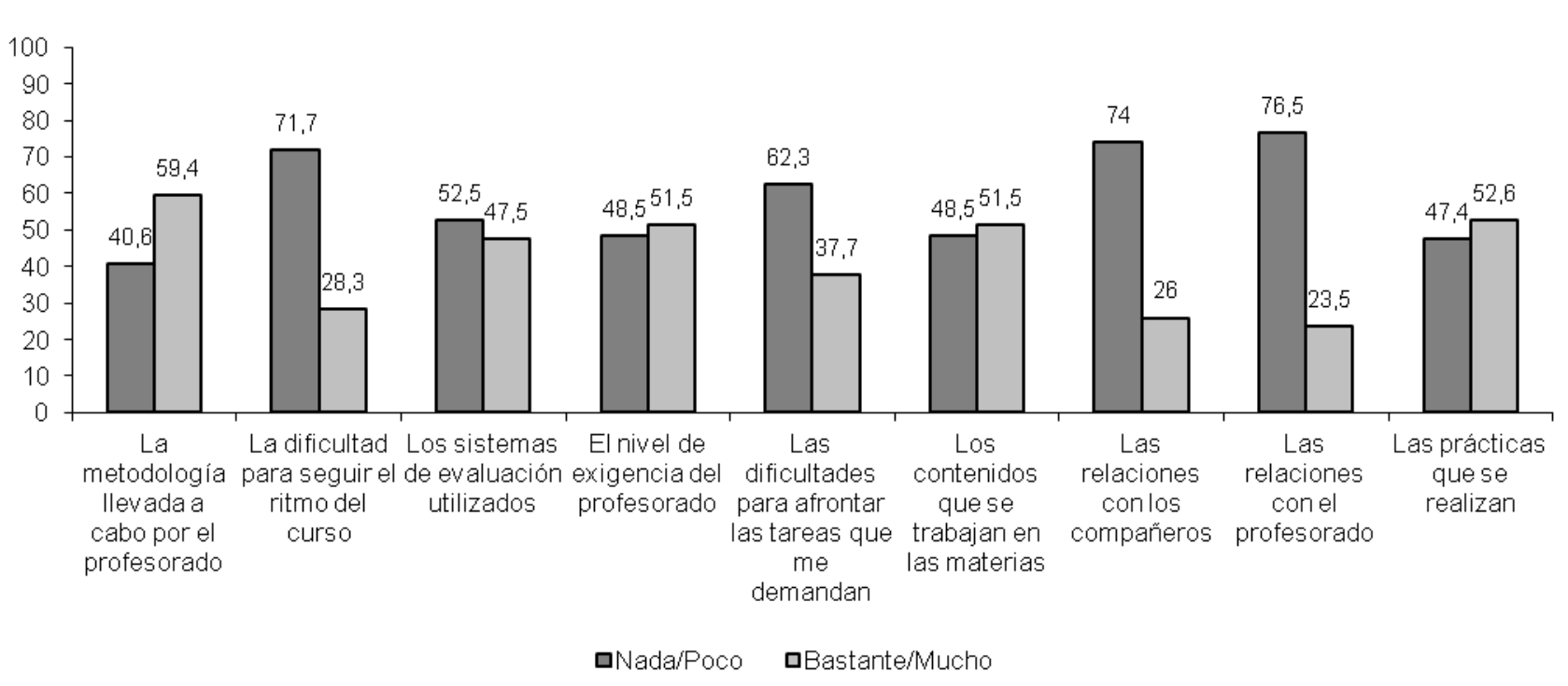

Fuente: elaboración propia

Los datos analizados han puesto de manifiesto que la información que tenían los estudiantes sobre la universidad en el momento de acceso a los estudios, estaba referida principalmente a las salidas profesionales de la carrera $(\bar{x}=3,35)$, los contenidos de las asignaturas $(\bar{x}=3,24)$ y la estructura curricular del plan de estudios $(\bar{x}=3,12)$. En menor medida, se preocuparon por conocer las competencias que se pretendía desarrollar a lo 
largo del título $(\bar{x}=2,62)$ y los departamentos implicados en impartir docencia en la carrera $(\bar{x}=2,34)$.

\subsection{Proceso formativo}

A nivel general, y a pesar de que tan solo un $17,7 \%$ estudiaba diariamente o los días previos al examen (20,4\%), los estudiantes manifestaban preocupación por su proceso formativo, dado que acudían a clase regularmente (90,4\%), invertían entre 4 y 12 horas semanales de estudio $(65,2 \%)$ y consideraban tener un conocimiento general del perfil profesional de la carrera (77,9\%). Esta preocupación por el estudio quedaba reflejada al indicar que poseían un método específico para organizar su tiempo $(\bar{x}=3,09)$. Esta continuidad y constancia en el estudio se veía proyectada en el proceso de aprendizaje de los estudiantes, en tanto eran estudiantes que participaban activamente en las clases presenciales $(\bar{x}=2,97)$, para aportar sugerencias o resolver dudas $(\bar{x}=2,90)$ que revertirían positivamente en la planificación de su carrera académica.

En consonancia con estos datos, los estudiantes sentían interés por recuperar aquellas clases a las que no habían podido acudir, principalmente solicitando ayudas y orientaciones a los compañeros (94,2\%), acudiendo a tutorías con el profesorado (34,6\%), dedicando más horas a preparar las asignaturas a las que no acudían $(22,1 \%)$ y en menor medida, preparando la materia de manera autónoma, a través del uso de la bibliografía recomendada por el profesor (15,4\%). Respecto a las relaciones esperadas entre la valoración de la carrera y las horas dedicadas al estudio (ítems 8-15) no se observó relación significativa $(p=, 574)$. Tampoco se apreció relación entre motivación y horas de estudio $(p=, 451)$ (ítems 10-15).

En lo que respecta a la valoración del profesorado universitario, los estudiantes opinaron que: respondía a las consultas y dudas en relación con su proceso formativo (63,8\%), estaba al día en las asignaturas en las que impartía docencia (45,7\%), tenía una buena relación con los estudiantes $(42,5 \%)$ y se preocupaba porque mantuvieran una actitud activa y participativa en el aula (45,0\%). No obstante, y en cuanto al proceso formativo, resaltaron la poca sensibilidad que tenía para que sus estudiantes entendieran las explicaciones de las materias (26,3\%), y lo poco claras que eran sus clases $(25,0 \%)$.

A pesar de esta realidad, el estudiantado coincidió en la idea de que a lo largo de su formación universitaria fue capaz de adquirir una serie de competencias que le servirían para su futuro desarrollo profesional. En este sentido, y tal y como se recoge en el Gráfico 3, los 
estudiantes se autodefinieron como competentes para el trabajo en equipo, la responsabilidad en el trabajo, las relaciones sociales, la toma decisiones, el pensamiento crítico y creativo, etc.

Gráfico 3. Competencias que dominan los estudiantes universitarios.

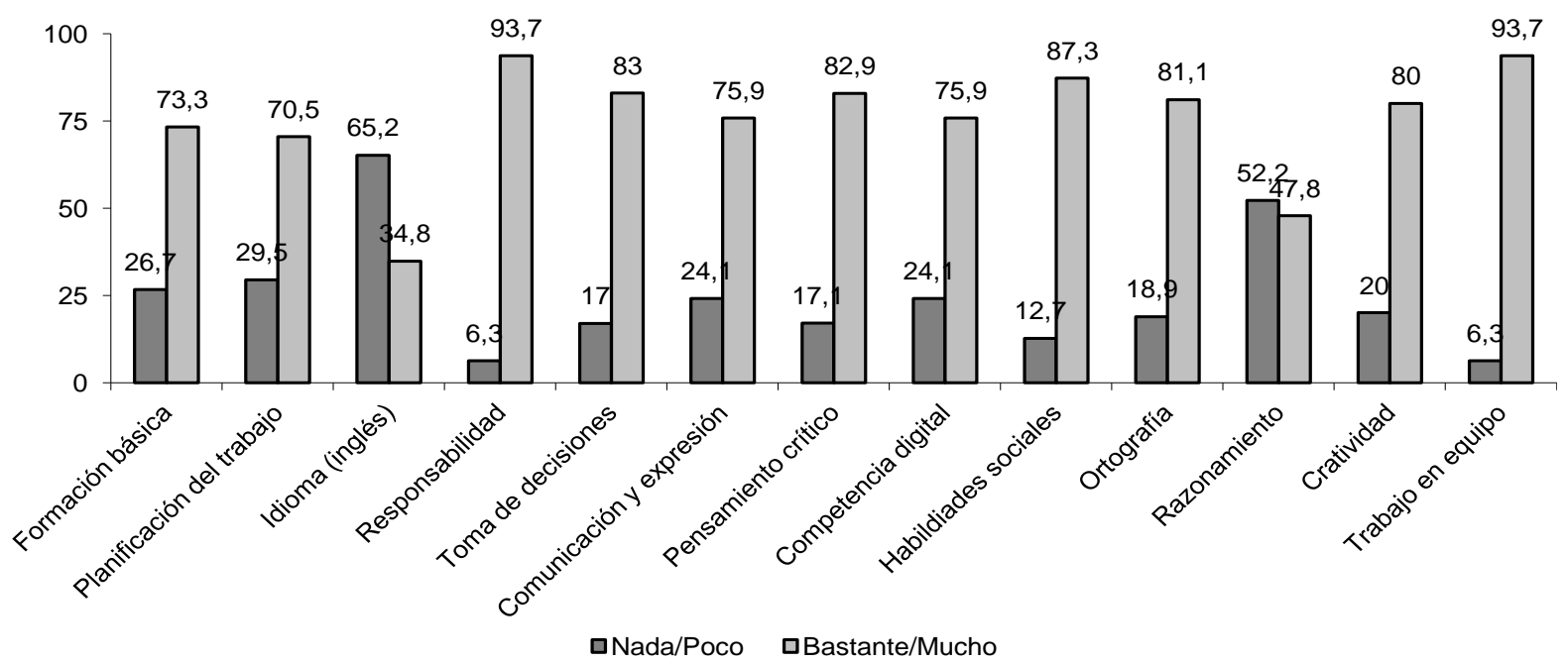

Fuente: elaboración propia

En relación con estos datos, el estudiantado no solo valoró que estaba adquiriendo estas competencias en el transcurso de la carrera universitaria, sino que además consideraba necesario adquirir una serie de habilidades de especial relevancia para ir configurando y planificando su proyecto académico-profesional. Así, resultaba importante: planificar su itinerario formativo en el transcurso de la carrera en función de sus objetivos personales $(88,3 \%)$, tomar decisiones y resolver problemas $(97,3 \%)$, tener iniciativa y capacidad emprendedora $(94,5 \%)$, asumir responsabilidades $(98,2 \%)$ y planificar el trabajo de clase $(98,2 \%)$. En esta misma línea, los estudiantes manifestaron la falta de asesoramiento e información acerca de la utilidad de los contenidos y las competencias que se debían desarrollar a lo largo de la carrera, puesto que "nadie me lo ha explicado" (p77), lo que dificultaba que tuvieran una visión de prospectiva sobre su carrera académica y profesional. 
Para conseguir este objetivo de planificación y gestión de la carrera académica, la estrategia de la tutoría universitaria se configura como un potente recurso que ayuda al estudiantado en este proceso de construcción personal y profesional. En este sentido, y a nivel general, este recurso de apoyo y asesoramiento fue poco utilizado y valorado por parte de los estudiantes. Tan solo el 38,4\% tenía conocimiento de la existencia de un plan de tutorías en su centro o facultad, del que solo acudía a este, una tercera parte. Del mismo modo, un bajo porcentaje de estudiantes $(31,0 \%)$ acudía regularmente a las tutorías de las asignaturas, para "resolver dudas" (p2) y para "revisión de exámenes" (p114), mientras que los que no acudían, argumentaban que era debido a la "falta de tiempo y porque coinciden en horas lectivas" (p17), o porque "no les veo utilidad" (p115).

\subsection{Metas y proyectos de futuro}

Una de las metas básicas que esperaba conseguir el alumnado con la carrera que cursaban, tenía que ver con "tener la formación adecuada" (p1), "una buena formación" (p6), y "un buen trabajo donde me sienta bien" (p76). Estas metas estaban íntimamente vinculadas con la planificación que hacían al finalizar sus estudios universitarios. Así, el $63,4 \%$ del alumnado tenía planificado los pasos que seguiría al finalizar los estudios, sobre todo centrados en "hacer otra carrera" (p95) o "seguir formándome hasta que consiga trabajar en lo que me gusta" (p38). Estas altas expectativas hacia los procesos de formación continua, se vieron reflejadas en su dedicación a los estudios, dado que consideraban que podrían finalizar su proceso de aprendizaje en el número de años que duraba la carrera (78,6\%), mientras que aquellos que creían que no lo conseguirían, sería principalmente debido a "motivos laborales" (p19), o porque "no dispongo de todo el tiempo" (p99). Del mismo modo, sentían interés por su futuro desarrollo profesional, informándose sobre las salidas laborales que tenía la carrera que cursaban (75,5\%), aunque aun tenían la percepción de "falta de información" (p41) y "algunas dudas" (p18) al respecto (Gráfico 4). 
Gráfico 4. Metas de los estudios universitarios

$100,00 \%$

$75,00 \%$

$68,50 \%$

$50,00 \%$

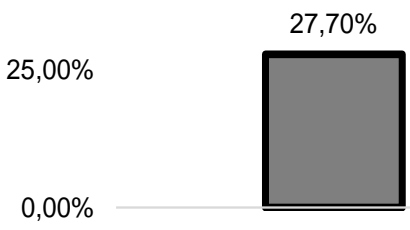

Formación

Fuente: elaboración propia

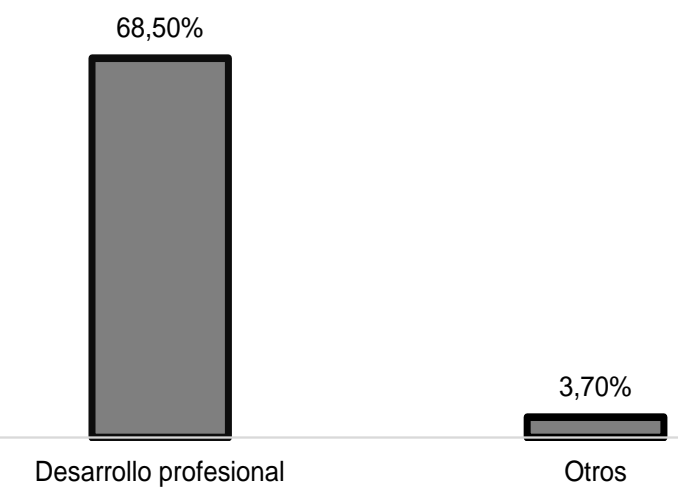

\section{Conclusiones}

Los resultados obtenidos en esta investigación ponen de manifiesto que la capacidad de adaptación a la educación superior, la orientación e información recibida, las características de la enseñanza y las expectativas de futuro, son factores claves que intervienen en los procesos de planificación y gestión de los proyectos formativos y profesionales del alumnado universitario. Al igual que en otros estudios realizados, en los que se aborda la formación del alumnado universitario (Mosteiro y Porto, 2000; Sánchez, 2001), los resultados de este trabajo indican que la elección de los estudios viene determinada por motivos relacionados con el interés por la carrera y las expectativas de desarrollo profesional. Desde esta perspectiva, cabe pensar que mucho del estudiantado, al decantarse por una carrera determinada, han pensado en los pasos o fases que deberán recorrer para alcanzar las metas académicas y profesionales que más les interesan.

El estudiante o la estudiante que accede a los estudios superiores con este perfil y con una idea clara de lo que quiere conseguir, es el que gestionará de manera más adecuada su proyecto formativo y profesional, que estará definido precisamente por las metas que desea alcanzar. Por el contrario, aquellos que se incorporan sin una idea clara de hacia dónde van y sin objetivos concretos por alcanzar, es decir, sin una planificación de su proyecto personal, son los más propensos a verse implicados en situaciones de fracaso académico. Esto explica, por ejemplo, que el $22,21 \%$ de estudiantes de la rama de Artes y Humanidades que ingresó en la Universidad de la Laguna en el curso 2010/11, no continuara sus estudios 
en el curso 2011/12. Esta falta de previsión y de planificación suele venir asociada a otras actitudes respecto al proceso formativo, como pueden ser una inadecuada organización y gestión del tiempo, escasa dedicación sistemática al estudio, escasa autoexigencia, actitud pasiva en el aula, escaso compromiso, etc.

No cabe duda de que el asesoramiento y la orientación al alumnado a lo largo de las distintas etapas de su desarrollo, constituyen un factor importante, el cual influirá en sus expectativas futuras, en la selección madura de los estudios por cursar y en la manera en la que organizan su proceso formativo. En este sentido, una de las conclusiones que se puede extraer de los resultados obtenidos y que destaca también Romero (2004), es que los procesos de exploración y de búsqueda de información son fundamentales en la construcción y planificación académica y profesional. Aquellos estudiantes que reciben una información adecuada, que participan en actividades exploratorias y de clarificación de expectativas y opciones académicas para definir sus intereses y planificar su itinerario, tendrán más facilidades para la adaptación y promoción en los estudios universitarios. Asimismo, estudiantes que acceden a la universidad con información clara y concreta, tendrán expectativas más ajustadas a la realidad, y una mayor definición de los pasos que deberán recorrer en su itinerario de aprendizaje, frente a aquellos que no han tenido una adecuada orientación. El estudiantado que elige los estudios en el último momento, que se matriculan en carreras que no son de su preferencia, o que se matriculan más bien porque tienen que elegir algo y no por cumplir con un proyecto personal definido, estarán más abocados a problemas de rendimiento, insatisfacción y fracaso.

Los datos obtenidos en el estudio confirman que hay un grupo importante del estudiantado universitario interesado y preocupado por su proceso formativo, que dedican tiempo y esfuerzo al aprendizaje y que evidencian un alto grado de motivación hacia la concesión de metas y el desarrollo de las competencias asociadas al perfil profesional. Y es que la universidad actual, tal como han señalado García et al. (2005), García y Salmerón (2010) y Villa y Poblete (2010), demanda un perfil de estudiantado con capacidad para planificar y gestionar un proyecto formativo que les capacite para el futuro desarrollo profesional. Así, un estudiante que asista a clase regularmente, que sienta motivación por los estudios (Gámez y Marrero, 2003), que se ajuste a las exigencias de la carrera, que esté satisfecho con la formación elegida, que asuma el esfuerzo personal en favor de los logros futuros y que gestione a largo plazo su proyecto académico y profesional (Álvarez, Cabrera, 
González y Bethencourt, 2006), tendrá muchas posibilidades de tener éxito en los estudios y lograr sus metas profesionales.

El proceso de atención, apoyo y orientación, no debe concluir en los primeros momentos o una vez que el estudiante accede a la carrera, sino mantenerse a lo largo de esta, hasta el momento cuando afronta la transición al mercado de trabajo. $Y$ una de las estrategias claves que ha cobrado gran relevancia para llevar a cabo este proceso de atención personalizada y apoyo al proceso de aprendizaje autónomo del alumnado, es la tutoría. Sin embargo, llama la atención el poco uso que los participantes en este estudio hacían de la tutoría y el desconocimiento que tenían acerca de la misma. Cambiar las concepciones previas que acerca de la tutoría y del tutor tienen los alumnos y las alumnas que acceden a los estudios universitarios, integrar la tutoría como un elemento activo y facilitador del proceso de aprendizaje del alumnado, ofrecer desde la tutoría estrategias y recursos para el desarrollo de competencias que contribuyan al desarrollo integral del alumnado, y guiarles en el proceso de elaboración de su proyecto personal, es uno de los retos que tiene planteados la institución universitaria.

\section{Referencias}

Alonso, Miguel Aurelio. (2004). Centralidad del trabajo y metas en el trabajo: dos variables claves en orientación laboral. Revista de Educación, 335, 319-344.

Álvarez, Manuel, Figuera, Pilar y Torrado, Mercedes. (2011). La problemática de la transición bachillerato-universidad en la Universidad de Barcelona. Revista Española de Orientación y Psicopedagogía, 22(1), 15-27.

Álvarez, José Ángel, Duque, Esther, García, Manuel, García, María Valme, García, Concepción, Ory, Ignacio y Rodríguez, Irene. (2004). Causas de la falta de asistencia a clase en una Facultad de Ciencias: análisis de los resultados de encuestas personales. Revista Española de Orientación y Psicopedagogía, 15(2), 317-335.

Álvarez, Pedro. (2002). La función tutorial en la universidad; una apuesta por la mejora de la calidad de la enseñanza. Madrid: EOS.

Álvarez, Pedro y López, David. (2011). El absentismo en la enseñanza universitaria: un obstáculo para la participación y el trabajo autónomo del alumnado. Bordón, 63(3), 4154.

Álvarez, Pedro, Cabrera, Lidia, González, Miriam y Bethencourt, José. (2006). Causas del abandono y prolongación de los estudios universitarios. Paradigma, 27(1), 7-36.

Álvarez, Pedro (coord.) (2012). Tutoría universitaria inclusiva. Madrid: Narcea. 
Arenas, Mar, Bilbao, Amelia, Rodríguez, María Victoria y Jiménez, Mariano. (2003). Matemáticas en los estudios de Economía y Gestión de Empresas en el marco de los acuerdos de Bolonia. Actas de las XI Jornadas de ASEPUMA, Oviedo.

Blanco, Ángeles. (2009). Desarrollo y evaluación de competencias en educación superior. Madrid: Narcea.

Cabrera, Lidia, Bethencourt, José, González, Miriam y Álvarez, Pedro. (2006). Un estudio transversal retrospectivo sobre prolongación y abandono de estudios universitarios. RELIEVE, http://www.uv.es/RELIEVE/v12n1/RELIEVEv12n1 1.pdf

Recuperado de

Cano, Rufino. (2009). Tutoría universitaria y aprendizaje por competencias, ¿Cómo lograrlo? REIFOP, 12(1), 181-204.

Castro, Alejandro y Sánchez, María del Pilar. (2000). Objetivos de vida y satisfacción autopercibida en estudiantes universitarios. Psicothema, 12(1), 87-92.

Coriat, Moisés y Sanz, Rafael. (2005). Orientación y tutoría en la Universidad de Granada. Granada: Editorial Universidad de Granada.

Corominas, Enrie e Isus, Sofía. (1998). Transiciones y orientación. Revista de Investigación Educativa, 16(2), 155-184.

Corominas, Enrie. (2006). Nuevas perspectivas de la orientación profesional para responder a los cambios y necesidades de la sociedad de hoy. Revista Estudios sobre Educación, (11), 91-110.

Davia, María Ángeles. (2004). La inserción laboral de los jóvenes en la Unión Europea: un estudio comparativo de trayectorias laborales. Madrid: Consejo Económico y Social de España.

Echeverría, Benito (coord.). (2008). Orientación profesional. Barcelona: UOC.

Elias-Andreu, Marina. (2008). Los abandonos universitarios: retos antes del Espacio Europeo de Educación Superior. Revista Estudios Sobre Educación, (15), 101-121.

España. (2007). Real Decreto 1393/2007, de 29 de octubre, por el que se establece la ordenación de las enseñanzas universitarias oficiales. Boletín Oficial del Estado, 30 de octubre 2007, 260, pp. 44037-44048.

España. (2010). Real Decreto 861/2010, de 2 de julio, por el que se modifica el Real Decreto 1393/2010, de 29 de octubre, por el que se establece la ordenación de las enseñanzas universitarias oficiales. Boletín Oficial del Estado, 3 de julio 2010, 161, pp. 5845458468.

España. (2010). Real Decreto 1791/2010, de 30 de diciembre, por el que se aprueba el Estatuto del Estudiante Universitario. Ministerio de Educación. Boletín Oficial del Estado, 31 de diciembre 2010, 318, pp. 109353-109380. 
Figuera, Pilar, Torrado, Mercedes y Dorio, Inmaculada. (2011). La integración académica y social en la Universidad: la importancia de las redes sociales. XV Congreso Nacional y I Internacional de Modelos de Investigación Educativa.

Gairín, Joaquín, Figuera, Pilar y Triadó, Xavier (Eds.). (2010). L’abandonament del sestudiants a les universitats catalanes. Barcelona: AQU.

Gallego, Sofía. (1999). Cómo planificar el desarrollo profesional; actividades y estrategias de autoorientación. Barcelona: Laertes.

Gámez, Elena y Marrero, Hipólito. (2003). Metas y motivos en la elección de la carrera universitaria: un estudio comparativo entre psicología, derecho y biología. Anales de Psicología, 19(1), 121-131.

García, Narciso, Asensio, Inmaculada, Carballo, Rafael, García, Mercedes y Guardia, Soledad. (2005). La tutoría universitaria ante el proceso de armonización europea. Revista de Educación, (337), 89-210.

García, Narciso. (2008). La función tutorial de la universidad en el actual contexto de la educación superior. Revista Interuniversitaria de Formación de Profesorado, 22(1), 21 48.

García, Catalina y Salmerón, Román. (2010). Adaptación de la metodología al Espacio Europeo de Educación Superior. Análisis de la opinión de los alumnos. Revista Estudios Sobre Educación, (19), 237-260.

González, Miriam, Álvarez, Pedro, Cabrera, Lidia y Bethencourt, José. (2007). El abandono de los estudios universitarios: factores determinantes y medidas preventivas. Revista Española de Pedagogía, 65(236), 71-85.

Guichard, Jean. (1995). La escuela y las representaciones de futuro de los adolescentes. Barcelona: Laertes.

Hernández, Víctor. (2004). Expectativas vocacionales de resultados en estudiantes de secundaria. Revista de Investigación Educativa, 22(1), 89-112.

Martín, Mercedes, Moreno, Emilia y Padilla, Teresa. (1998). La orientación para el acceso a la universidad: análisis de las necesidades expresadas por una muestra de estudiantes de nuevo ingreso. Revista Española de Orientación y Psicopedagogía, 9(16), 257-271.

Martínez, Pilar. (2003). La importancia de la toma de decisiones. En AA.VV: Guía de salidas profesionales. Titulaciones de la Universidad de Murcia (pp. 234-236). Murcia: COIE.

Martínez, Marius. (2009). La orientación y la tutoría en la universidad en el marco del Espacio Europeo de Educación Superior (EEES). Revista Fuentes, 9, 78-97.

Mendías, Ana María y Camacho, Salvador. (2004). Motivos que impulsan a los alumnos a estudiar magisterio. El caso de la Universidad de Granada. Revista de Educación de la Universidad de Granada, (17), 91-107. 
Monge, Juan José. (1993). Actitud de los alumnos de magisterio de la Universidad de Cantabria hacia la profesión docente al comienzo y final de la carrera. Revista Interuniversitaria de Formación del Profesorado, (16), 87-96.

Mosteiro, María Josefa y Porto, Ana María. (2000). Los motivos de elección de estudios en alumnos y alumnas de universidad. Innovación Educativa, (10), 121-132.

Peter, Jarvis. (2006). Universidades corporativas: nuevos modelos de aprendizaje en la sociedad global. Madrid: Narcea.

Rivas, Francisco. (1990). La elección de los estudios universitarios. Madrid: Consejo de Universidades.

Rodríguez, María Luisa. (1998). Enseñar y aprender a tomar decisiones vocacionales en la ESO. Barcelona: Laertes.

Rodríguez, María Luisa. (2003). Cómo orientar hacia la construcción del proyecto profesional. Autonomía individual, sistema de valores e identidad laboral de los jóvenes. Bilbao: Desclée Brouwer.

Romero, Soledad. (2003). La construcción de proyectos profesionales y vitales: aplicación de la orientación a personas en centros de formación y en busca de su primer empleo. Bordón, 55(3), 425-432.

Romero, Soledad. (2004). Aprender a construir proyectos profesionales y vitales. Revista Española de Orientación y Psicopedagogía, 15(2), 337-354.

Romero, Soledad. (2009). El proyecto profesional y vital. En Luis Sobrado y Alejandra Cortés (coords.), Orientación Profesional. Nuevos escenarios y perspectivas (pp. 111-119). Barcelona: Biblioteca Nueva.

Ryan, Michael y Glenn, Patricia. (2003). Increasing one-year retention rates by focusing on academic competence: An empirical odyssey. Journal of College Student Retention, 4(3), 297-324.

Salinas, Jesús. (2000). El rol del profesorado en el mundo digital. En Lluís del Carmen (ed.), Simposio sobre la formación inicial de los profesionales de la educación (pp. 305-320). Barcelona: Universitat de Girona

Sánchez, María Fé. (2001). La orientación universitaria y las circunstancias de elección de los estudios. Revista de Investigación Educativa, 19(1), 39-61.

Sánchez, María Fé, Guillamón, José Rafael, Ferrer, Paula, Villalba, Encarnación, Martín, Ana y Pérez, Juan Carlos. (2008). Situación actual de los servicios de Orientación Universitaria: un estudio descriptivo. Revista de Educación, (345), 329-352.

Santana, Lidia (coord.). (2001). Trabajo, educación y cultura; un enfoque interdisciplinar. Madrid: Pirámide. 
Santana, Lidia y Feliciano, Luis. (2009). Dificultades en el proceso de toma de decisiones académico-profesionales: el reto de repensar la orientación en Bachillerato. Revista de Educación, (350), 323-350.

Sola, Tomás y Moreno, Antonio. (2005). La acción tutorial en el contexto del Espacio Europeo de Educación Superior. Educación y Educadores, 8, 123-143.

Taveira, María y Rodríguez, María Luisa. (2010). La gestión personal de la carrera y el papel de la orientación profesional. Teoría, práctica y aportaciones empíricas. Revista Española de Orientación y Psicopedagogía, 21(2), 335-345.

Taylor, Janice y Millar, Thedore. (2002). Necessary components for evaluating minority retention programs. NASPA Journal, 39(3), 266-282.

Tejada, Carlos. (2003). Perfil, motivaciones y expectativas de los alumnos de primer curso de la Escuela Universitaria de Biblioteconomía y Documentación de la Universidad Complutense de Madrid. Revista General de Información y Documentación (1), 195211.

Vieira, María José. (2008). Criterios para la evaluación del sistema de apoyo y orientación al estudiantado universitario: revisión y propuestas. Revista de Educación, (345), 399423.

Villa, Aurelio y Poblete, Manuel. (2010). Aprendizaje basado en competencias. Bilbao: Mensajero.

Zabalza, Miguel Ángel. (2003). Competencias docentes del profesorado universitario: calidad y desarrollo profesional. Madrid: Narcea. 